\title{
Type II Fusarium head blight susceptibility factor identified in wheat
} Hales, B. ${ }^{1}$, Steed, A. ${ }^{1}$, Giovannelli, V. ${ }^{1}$, Burt, C. ${ }^{1}$, Lemmens, M., ${ }^{2}$ Molnár-Láng, M. ${ }^{3}$, Nicholson, P. ${ }^{1 *}$

${ }^{1}$ Department of Crop Genetics, John Innes Centre, Norwich Research Park, Norwich, NR4 7UH, England

2 University of Natural Resources and Life Sciences, Institute for Biotechnology in Plant Production, Department of Agrobiotechnology, IFA Tulln, A-3430 Tulln, Austria

${ }^{3}$ Agricultural Institute, Centre for Agricultural Research, H-2462 Martonvásár, Hungary

* Correspondence: paul.nicholson@jic.ac.uk, 441603450616

Word count: 5787

Submission date: 06/02/2020

Number of tables: 3

Number of figures: 8 


\section{Short title}

Type II Fusarium head blight susceptibility factor identified in wheat

\section{Highlight}

We have identified a Type II Fusarium head blight susceptibility factor on the short arm of wheat chromosome 4D and refined its position to a $31.7 \mathrm{Mbp}$ interval.

\section{Abstract}

Fusarium head blight (FHB) causes significant grain yield and quality reductions in wheat and barley. Most wheat varieties are incapable of preventing FHB spread through the rachis, but disease is typically limited to individually infected spikelets in barley. We point inoculated wheat lines possessing barley chromosome introgressions to test whether FHB resistance could be observed in a wheat genetic background. The most striking differential was between $4 \mathrm{H}(4 \mathrm{D})$ substitution and $4 \mathrm{H}$ addition lines. The $4 \mathrm{H}$ addition line was similarly susceptible to the wheat parent, but the $4 \mathrm{H}(4 \mathrm{D})$ substitution line was highly resistant, which suggests that there is an FHB susceptibility factor on wheat chromosome 4D. Point inoculation of Chinese Spring 4D ditelosomic lines demonstrated that removing 4DS results in high FHB resistance. We genotyped four Chinese Spring 4DS terminal deletion lines to better characterise the deletions in each line. FHB phenotyping indicated that lines del4DS2 and del4DS-4, containing smaller deletions, were susceptible and had retained the susceptibility factor. Lines del4DS-3 and del4DS-1 contain larger deletions and were both significantly more resistant, and hence had presumably lost the susceptibility factor. Combining the genotyping and phenotyping results allowed us to refine the susceptibility factor to a $31.7 \mathrm{Mbp}$ interval on 4DS.

\section{Key Words}

Fusarium, scab, susceptibility, wheat, barley, aneuploid, deletion

\section{Abbreviations}

Days post inoculation (dpi)

Deoxynivalenol (DON)

DON-3-O-glucoside (D3G)

Ditelosomic (DT)

Fusarium head blight (FHB)

Fraction length (FL)

Quantitative trait locus (QTL)

UDP-glucosyltransferase (UGT) 
1 Introduction

2 Fusarium head blight (FHB) is an economically important fungal disease of various

3 cereal crop species, in particular wheat (Triticum aestivum) and barley (Hordeum

4 vulgare). In wheat, the primary symptom is the premature bleaching of spikelets that

5 progressively spreads through the head. Infected spikelets produce shrivelled and

6 chalky grain, which can have a significant impact on yield. Furthermore, mycotoxins

7 accumulate in infected grain, which are harmful to humans and animal consumers.

8 The most important mycotoxin is deoxynivalenol (DON) which acts as a virulence

9 factor in wheat by promoting the spread of the fungus (Bai et al., 2002; Langevin et

10 al., 2004). Fusarium graminearum and F. culmorum are the most prevalent species

11 responsible for FHB. Both species are capable of producing large quantities of DON

12 (Scherm et al., 2013) and hence tend to be the most aggressive pathogens of wheat.

13 Resistance to initial infection (Type I) and to the spread of infection through the

14 rachis (Type II) were first proposed by Schroeder and Christensen (1963) and

15 remain the two most widely considered forms of resistance. Numerous small-effect

16 Type II and fewer Type I FHB quantitative trait loci (QTL) have been reported and

17 are reviewed by Buerstmayr et al. (2009) and more recently by Buerstmayr et al.

18 (2019). In addition to these two main types of FHB resistance, there is resistance to

19 kernel infection (Type III), host tolerance to FHB and/ or DON (Type IV) and

20 resistance to the accumulation of DON (Type V) (Boutigny et al., 2008; Gunupuru et

21 al., 2017). Single amino acid changes to the DON target, ribosomal protein L3

22 (RPL3), have been demonstrated to improve tolerance to DON in yeast and hence

23 this is a possible target to improve type IV resistance (Lucyshyn et al., 2007;

24 Mitterbauer et al., 2004). Type $\mathrm{V}$ resistance is commonly considered to be a

25 component of Type II resistance, as it typically limits disease spread (Gunupuru et

26 al., 2017), and can be subdivided into Class 1 : processes that chemically modify

27 DON to a less toxic form, and Class 2: processes that prevent the accumulation of

28 DON and other trichothecene mycotoxins (Boutigny et al., 2008). The most widely

29 reported form of host detoxification of DON is by UDP-glucosyltransferase (UGT)

30 proteins, which glucosylate DON to the less toxic DON-3-O-glucoside (D3G)

31 (Poppenberger et al., 2003). More recent studies have identified other pathways

32 capable of detoxifying DON. For example, bacterial aldo-keto reductases were 
33 demonstrated to be involved in epimerising DON to 3-epi-DON (Hassan et al., 2017;

34 He et al., 2017).

35 Wheat and barley differ noticeably in Type II resistance. Wheat typically possesses

36 some degree of Type II susceptibility whilst, in contrast, barley is generally highly

37 resistant to fungal spread through the rachis (Langevin et al., 2004). Furthermore,

38 whilst DON has been shown to function as a virulence factor in wheat (Langevin et

39 al., 2004), DON does not appear to possess such a role during infection of barley

40 heads (Maier et al., 2006).

41 The reasons for this marked difference in Type II susceptibility of wheat and barley

42 are not well understood. Defined genetic stocks of wheat containing all or part of

43 barley chromosomes offers an insight into which barley chromosomes contribute

44 most strongly to Type II FHB resistance and whether this resistance can be

45 expressed, and potentially utilised, in a wheat genetic background. Herein, we report

46 on a series of experiments to establish whether this difference in FHB susceptibility

47 is because barley carries genes conferring resistance, wheat carries genes

48 conferring susceptibility, or whether it is a combination of both factors. Following this,

49 we investigated the location of a major effect identified on wheat chromosome 4D

50 that appears to significantly compromise resistance to disease spread through the

51 rachis (Type II resistance).

52 To date, there have been few reports of FHB susceptibility factors. Garvin et al.

53 (2015) identified a spontaneous deletion of a portion of the long arm of 3D, which

54 appeared to be responsible for increased FHB resistance, suggesting that the

55 deleted region carries an FHB susceptibility factor in the cultivar Apogee. Ma et al.

56 (2006) point inoculated the existing ditelosomic lines of Chinese Spring that each

57 lack individual chromosome arms. They found that the loss of individual

58 chromosome arms can improve, as well as compromise, FHB resistance (Ma et al.,

59 2006). Their data suggested that some chromosome arms, especially 7AS, 3BL,

60 7BS and 4DS, are likely to contain FHB susceptibility factors (Ma et al., 2006).

61 Although the gene(s) underlying Fhb1, the most widely deployed FHB resistance

62 QTL, remains controversial, there is evidence that Fhb1 may be considered a

63 disrupted susceptibility factor (Su et al., 2019; Su et al., 2018). Plant hormones play

64 an important role in responding to disease. Host response to FHB infection is 
65 particularly sensitive to disrupting phytohormone production or perception. Plants

66 insensitive to ethylene and brassinosteroid signalling exhibits increased FHB

67 resistance, suggesting that the fungus is exploiting such physiological processes

68 (Chen et al., 2009; Goddard et al., 2014). There is significant potential in identifying

69 and characterising susceptibility factors, with the aim of eliminating them from elite

70 cultivars to enhance resistance to FHB and other economically important diseases. 


\section{Materials and Methods}

\section{Plant material}

73 Wheat-barley addition, substitution and translocation lines were developed at the

74 Hungarian Academy of Sciences, Agricultural Institute, Centre for Agricultural

75 Research, Hungary (Table 1). An independent set of wheat-barley addition lines, of

76 the wheat variety Chinese Spring and the barley donor variety Betzes, were

77 generated by Islam et al. (1981) and obtained from the Genetic Resources Unit at

78 the John Innes Centre, Norwich, UK.

79 Chinese Spring and its 4D ditelosomic (DT) lines were acquired from the Germplasm

80 Resource Unit, John Innes Centre, Norwich, UK. The lines DT(4DL) and DT(4DS)

81 lack 4DS and 4DL, respectively. Four homozygous Chinese Spring terminal deletion

82 lines of 4DS, described by Endo and Gill (1996), were obtained from Kansas State

83 University, USA. The lines acquired were $4532 \mathrm{~L} 1$ ( $F L=0.53), 4532 \mathrm{~L} 2(\mathrm{FL}=0.82)$,

$844532 \mathrm{L3}(\mathrm{FL}=0.67)$ and $4532 \mathrm{~L} 4(\mathrm{FL}=0.77)$, henceforth referred to as del4DS-1,

85 del4DS-2, del4DS-3 and del4DS-4, respectively.

\section{Marker development and genotyping}

87 Homoeologue nonspecific markers were designed to simultaneously amplify

88 fragments of homoeologous genes on $4 A, 4 B$ and 4D. Sequence information of 4D

89 genes and corresponding homoeologous genes were obtained from Ensembl Plants

90 (http://plants.ensembl.org/Triticum aestivum/Info/Index). Gene names and the

91 physical positions reported correspond to the IWGSC RefSeq v1.1 wheat genome

92 assembly (IWGSC, 2018). Sequence insertions and deletions (indels) between

93 homoeologous gene sequences were exploited to enable distinction of the three

94 resulting PCR products. Forward primers were M13-tailed to enable incorporation of

95 a fluorescent adaptor to PCR products, as described by Schuelke (2000). 37

96 markers designed as such were used to characterise the deletions in four Chinese

97 Spring 4DS terminal deletion lines (Table 2).

98 DNA was extracted from freeze-dried leaf tissue as described by Pallotta et al.

99 (2003). PCR reactions were prepared using HotStarTaq Mastermix (Qiagen)

100 following the manufacturer's instructions and amplified using the following steps: 95

$101{ }^{\circ} \mathrm{C} 15 \mathrm{~min} ; 35$ cycles of: $95^{\circ} \mathrm{C} 1 \mathrm{~min}, 58^{\circ} \mathrm{C} 1 \mathrm{~min}, 72{ }^{\circ} \mathrm{C} 1 \mathrm{~min} ; 72^{\circ} \mathrm{C} 10 \mathrm{~min}$. PCR

102 products were separated using an ABI 3730xI DNA analyser (Applied Biosystems) 
103 and resolved using Peak Scanner 2 software (Applied Biosystems). Up to five

104 markers were multiplexed following PCR to increase assay efficiency.

105 Primers were designed to specifically amplify within a $5 \mathrm{H}$ barley UGT-

106 glucosyltransferase (HORVU5Hr1G047150), whilst avoiding amplification of wheat

107 orthologues (primer sequences: GATGAGGTTTGAGATTTGCGGA,

108 CACGAGCACAACAGATGAATTCA). PCR reactions were prepared using Taq

109 Mastermix (Qiagen) and amplified using the following PCR settings: $94^{\circ} \mathrm{C} 3 \mathrm{~min} ; 35$

110 cycles of: $94^{\circ} \mathrm{C} 30 \mathrm{sec}, 58^{\circ} \mathrm{C} 30 \mathrm{sec}, 72^{\circ} \mathrm{C} 1 \mathrm{~min} ; 72^{\circ} \mathrm{C} 10 \mathrm{~min}$. PCR products

111 were separated on a $0.8 \% \mathrm{w} / \mathrm{v}$ agarose gel.

\section{FHB evaluation and statistical analysis}

113 Highly virulent DON-producing isolates of $F$. graminearum or F. culmorum were used

114 in disease experiments. Production of inoculum was carried out as described

115 previously in Gosman et al. (2005). Wheat heads were inoculated at mid-anthesis.

116 The conidial suspension, adjusted to $1 * 10^{6}$ spores $\mathrm{ml}^{-1}$, was injected in to a spikelet

117 approximately central on the wheat head. The spread of disease symptoms was

118 scored regularly after inoculation. Polytunnel experiments were organised in a

119 randomised complete block design with four replicates each containing four or five

120 plants per line. For the glasshouse experiment, at least 16 plants per lines were

121 randomised and individual inoculated heads were considered as replicates.

122 Disease data were analysed using a linear mixed model (REML) in Genstat software

123 (v18.1) to assess the variation attributable to line (fixed), inoculation date (fixed), the

124 interaction between line and inoculation date (fixed), and replicate (random), where

125 factors were significant in the model. Data from which residuals were not normally

126 distributed or where residuals did not appear independent of fitted values were log10

127 transformed, which was sufficient in correcting for these assumptions. Predicted

128 mean and standard error values were calculated for lines included in the REML.

129 Pairwise comparisons were made between the wild type wheat parent/ genetic

130 background and the other genotypes tested in each experiment using Fisher's

131 protected least significant difference. All predicted values generated from

132 transformed data were back transformed to the original scale for presentation. 


\section{DON evaluation and statistical analysis}

134 DON was purified to $>98 \%$ at IFA-Tulln, as described by Altpeter and Posselt

135 (1994). DON application was carried out on wheat spikes at mid-anthesis, following a 136 protocol modified from Lemmens et al. (2005). Two adjacent spikelets opposite to 137 each other on the wheat head and approximately central on the head, were cut with 138 scissors approximately central on the spikelet. $1-2 \mathrm{~h}$ after cutting, $10 \mu \mathrm{L}$ of DON 139 solution (10 mg / mL amended with $0.01 \% \mathrm{v} / \mathrm{v}$ Tween 20 ) was applied to the two 140 outer florets of each cut spikelet, between the palea and lemma. To increase the 141 humidity at the site of DON application, treated wheat heads were bagged. At $48 \mathrm{~h}$ 142 post-application, the DON application was repeated, and heads bagged again. 143 Hence, each treated wheat head received a total application of $0.8 \mathrm{mg}$ DON. After a

144 further $48 \mathrm{~h}$, crossing bags were removed from the DON treated heads. The severity 145 of bleaching for each treated wheat head was scored, out of ten, daily between five 146 and nine days post application (from the first application). A score of zero was given 147 when no evidence of DON damage was present and a score of ten was recorded 148 when the spike was completely bleached above the point of DON application. Scores 149 between one and nine were used to record the progressive yellowing and bleaching 150 of the DON treated wheat heads, which occurred relatively uniformly above the point 151 of DON application in the case of Chinese Spring (Figure S1). After the experiment, 152 DON-treated and untreated heads from each plant were harvested. From each plant 153 with a DON treated head, a comparable untreated head (with similar spikelet number 154 and head length) was selected for grain weight analysis. Grain number and grain 155 weight data were collected from DON treated and comparable untreated heads from 156 each plant, to observe any difference in the effect of DON on grain filling.

157 DON bleaching data and associated grain data were analysed using a REML. Both 158 DON bleaching data and grain data were log10 transformed to achieve normality of 159 residuals and to ensure residuals were independent of fitted values. For bleaching 160 data, line was included as a fixed term and replicate as a random term in the model. 161 For DON grain data, the REML model was constructed using line, treatment (DON 162 treated or untreated heads), and the interaction between line and treatment as fixed 163 terms, and replicate as a random term. Ratios between mean treated and untreated 164 values were calculated by subtracting the predicted mean of log10 DON treated 165 heads from the predicted mean of log10 untreated heads for each line. Standard 
166 errors of predicted means were calculated as the square root of the sum of the

167 squared standard errors of the predicted mean values. The calculated mean and

168 standard error values were back transformed, resulting in the presentation of DON

169 treated/ untreated mean grain weight ratios for each line. 
170 Results

171 Effect of barley chromosome additions, substitutions, translocations 172 and centric fusions on type II FHB susceptibility in the winter wheat 173 variety Martonvasari 9 (Mv9kr1)

174 FHB point inoculation experiments of the wheat-barley material were conducted

175 twice and are described as experiment 1 (Figure 1A) and experiment 2 (Figure 1B)

176 henceforth. The experiments showed very similar results for most of the lines tested.

177 FHB symptoms were always restricted in both barley varieties, Igri and Betzes, and

178 did not spread from the inoculated spikelet. For this reason, Igri and Betzes were

179 only included as control lines in experiment 1 (Figure 1A). The primary wheat

180 parent, Mv9kr1, was susceptible to the spread of the fungus in both repeats of the 181 experiment.

182 The addition of barley chromosomes $2 \mathrm{H}(2 \mathrm{H}$ add) and $6 \mathrm{HS}(6 \mathrm{HS}$ add) appeared to 183 have no effect on FHB resistance in either experiment. Disease symptoms in these 184 lines were not statistically significantly different from that of Mv9kr1. The 6BS.6BL$1854 \mathrm{HL}$ translocation (6B-4H trans) was significantly more susceptible than Mv9kr1 ( $p<$ 1860.001 in both experiments). Whilst the 3HS.3BL centric fusion line (3HS.3BL centric) 187 was more highly susceptible in experiment $1(p<0.001)$, the line showed similar 188 disease to Mv9kr1 in experiment $2(p=0.566)$. The addition of chromosomes $1 \mathrm{HS}$ 189 (1HS add) and 7H (7H add), in addition to the 5HS-7DS.7DL wheat-barley 190 translocation (5H-7D trans) and the 2DS.2DL-1HS translocation line (2D-1H trans)

191 all showed highly significant increases in FHB resistance compared to Mv9kr1 ( $\mathrm{p}<$ 1920.001 in both experiments for all lines). The $3 \mathrm{H}$ addition ( $3 \mathrm{H}$ add) was inconsistent 193 between the two experiments. In experiment 1, the $3 \mathrm{H}$ addition was significantly 194 more susceptible to FHB than Mv9kr1 ( $p=0.004)$ whilst, in experiment 2 , it was 195 significantly more resistant $(p<0.001)$.

196 A particularly strong resistant phenotype was seen with the $4 \mathrm{H}(4 \mathrm{D})$ substitution, in 197 which disease was almost entirely restricted to the inoculated spikelet in both 198 experiments ( $p<0.001$ in both instances). In contrast to this, the addition of barley $1994 \mathrm{H}(4 \mathrm{H}$ add) showed similar disease levels to Mv9kr1 in experiment 1 ( $p=0.841$, 200 Figure 1A) and exhibited only a small increase in resistance in experiment $2(p=$ 2010.021 , Figure 1B). 
202 Effect of barley chromosome additions, substitutions, translocations 203 and centric fusions on type II FHB susceptibility in the spring wheat 204 variety Chinese Spring

205 An FHB point inoculation experiment was performed on wheat-barley addition lines 206 of the varieties Chinese Spring and Betzes, respectively (Figure 2). These lines 207 include addition lines of $5 \mathrm{HS}$ and $5 \mathrm{HL}$, which were absent in the lines generated in 208 the Mv9kr1 wheat background. As previously observed, Betzes showed almost no 209 disease spread from the inoculation point. Chinese Spring, on the other hand, 210 showed evidence of disease spread. FHB symptoms in the majority of addition lines 211 were not significantly different from Chinese Spring. The addition lines carrying the 212 barley chromosome arms 2HL, 6HS, 7HL and 7HS all showed significantly increased

213 FHB susceptibility compared to Chinese Spring.

214 The 5HL addition line exhibited significantly increased FHB resistance when

215 compared with Chinese Spring $(p<0.001)$, although the line was still significantly

216 more susceptible than Betzes $(p=0.042)$. The $5 \mathrm{HS}$ addition line was also statistically

217 significantly more resistant compared to Chinese Spring $(p=0.039)$. A marker

218 targeting the barley UDP-glucosyltransferase gene, HORVU5Hr1G047150,

219 confirmed that this gene was present in Betzes and the $5 \mathrm{HL}$ addition line, but was

220 absent in the 5HS addition line (Figure S2). Consistent with the previous

221 experiments, the $4 \mathrm{HL}$ and $4 \mathrm{HS}$ addition lines both showed similar FHB susceptibility 222 to Chinese Spring.

223 Type II FHB susceptibility and DON susceptibility in Chinese Spring 4D 224 ditelosomic lines

225 The contrast in the effect of adding $4 \mathrm{H}$ or substituting $4 \mathrm{D}$ with $4 \mathrm{H}$ indicated that the 226 presence of 4D may be responsible for a significant proportion of the susceptibility of 227 both Mv9kr1 and Chinese Spring. To test this possibility, Chinese Spring and two 228 ditelosomic lines: DT(4DL) and DT(4DS), missing 4DS and 4DL, respectively, were 229 tested in three independent FHB point inoculation experiments. Data is presented 230 here from a 2013 experiment conducted in a glasshouse, but the results were 231 replicated in a 2013 experiment under controlled conditions and in a polytunnel 232 experiment conducted in 2016. Chinese Spring and DT(4DS), missing 4DL, showed 233 very similar disease symptoms to each other (Figure 3). In contrast to this, DT(4DL), 
234 missing 4DS, was highly resistant to the spread of infection when compared to wild 235 type Chinese Spring $(p<0.001)$.

236 DON is widely believed to contribute towards Type II susceptibility by promoting the 237 spread of FHB. Hence, it is possible that the susceptibility factor may be responding 238 to DON and not the fungus itself. To confirm whether DON is involved, we applied 239 purified DON to wheat heads of Chinese Spring and two ditelosomic lines; DT(4DL) 240 and DT(4DS). Chinese Spring was moderately susceptible to DON, with an average 241 bleaching score of 3.39 (Figure 4A). DT(4DS), lacking 4DL, was not significantly 242 different from Chinese Spring (mean= 2.88; $\mathrm{p}=0.222$ ) (Figure 4A). On the other 243 hand, DT(4DL), lacking 4DS, was significantly more susceptible to DON induced 244 bleaching $($ mean $=7.64 ; p<0.001)$ (Figure 4A).

245 Grain was harvested and dissected from DON treated and untreated heads to 246 assess any difference in grain weight. These data closely mirrored the bleaching 247 data. Chinese Spring and DT(4DS) showed similar reductions in grain weight when 248 comparing DON treated and untreated heads (mean ratios of 0.522 and 0.506 , 249 respectively) (Figure 4B). In contrast, grain of DON treated DT(4DL) heads had a 250 proportionally much greater reduction in grain weight compared to untreated heads 251 (mean ratio= 0.290) (Figure 4B). The difference is evident when visually comparing 252 treated and untreated grain from the three lines; treated grain from DT(4DL) are 253 visibly smaller than those of Chinese Spring and DT(4DS) (Figure 4C).

254 These data suggest that DON is not implicated in the function of the susceptibility 255 factor. However, there does appear to be an independent DON resistance factor also 256 on 4DS.

\section{Precise characterisation of deletion sizes in Chinese Spring 4DS}

\section{8 terminal deletion lines}

259 Experiments using 4D ditelosomic lines strongly suggest that the FHB susceptibility 260 attributed to chromosome 4D is isolated to the short arm (4DS). Genotyping was 261 performed on four Chinese Spring lines with terminal deletions on 4DS to verify the 262 deletions present and more precisely position the deletion breakpoint in each line 263 relative to the physical map. Markers were designed that can reliably detect genes 264 on 4D and their homoeologues on 4A and 4B. The ability to detect and distinguish all 265 three homoeologues provides two internal positive controls for each marker when 
266 identifying deletions of any particular homoeologue. Up to five markers, tagged using 267 different fluorophores (NED, FAM, PET or VIC), were multiplexed into a single 268 sample for efficiency, using markers designed to produce PCR product sizes 269 sufficiently different for each gene target and its respective homoeologues when 270 resolved using capillary electrophoresis (Figure 5).

271 Genotyping was successful in identifying genes, and their respective physical 272 positions, flanking the deletion breakpoint in all four 4DS terminal deletion lines 273 (Table 3). A marker (BH0001) targeting the gene TraesCS4D02G001400 at the 274 extreme distal end of 4DS confirmed that all four lines were true terminal deletions.

275 The terminal deletion in del4DS-2 extends to between 50.6 and 51.6 Mbp. Line 276 del4DS-4 is deleted up to between 53.9 and $54.8 \mathrm{Mbp}$. The deletion in del4DS-3 277 ends between 83.3 and 85.6 Mbp. The deletion breakpoint in the largest terminal 278 deletion line, del4DS-1, ends between 111.1 and 140.9 Mbp.

\section{Chinese Spring 4DS terminal deletion lines and type II FHB susceptibility}

280 Euploid Chinese Spring and the four Chinese Spring 4DS terminal deletion lines 281 genotyped (del4DS-2, del4DS-4, del4DS-3 and del4DS-1, in ascending order of 282 terminal deletion size) were point inoculated in a polytunnel experiment in 2017 283 (Figure 6). Chinese Spring showed moderate levels of disease in this experiment, 284 with mean disease above the inoculation point of 1.84 bleached spikelets at 13 dpi. 285 Lines del4DS-2 $(p=0.796)$ and del4DS-4 $(p=0.278)$ showed similar disease levels to 286 that of euploid Chinese Spring (Figure 6 and Figure 7). Lines del4DS-3 and del4DS2871 both had significantly reduced disease with respect to euploid Chinese Spring $(p<$ 2880.001 for both lines) (Figure 6 and Figure 7).

289 This information was used to infer that the susceptibility factor was present in the two 290 deletion lines carrying the smaller deletions (del4DS-2 and del4DS-4) but was lost in 291 the two lines containing the larger deletions (del4DS-3 and del4DS-1). Hence, the 292 FHB susceptibility factor appears to reside between the deletion breakpoints of 293 del4DS-4 and del4DS-3; a 31.73 Mbp interval (Figure 8). 


\section{Discussion}

295 Previous studies have shown that barley is able to detoxify DON through

296 glucosylation by the UDP-glucosyltransferase UGT13248 (Schweiger et al., 2010).

297 This gene has been transgenically expressed in Arabidopsis where it was

298 demonstrated to increase resistance to DON (Schweiger et al., 2010). Furthermore,

299 expression of UGT13248 in wheat, under the maize ubiquitin promoter, increased

300 FHB resistance and transformants were demonstrated to more efficiently convert

301 DON to the less toxic DON-3-O-glucoside (Li et al., 2015). However, Xing et al.

302 (2018) demonstrated that overexpression of a wheat UGT-glucosyltransferase also

303 increased FHB resistance and reduced the DON concentration in grain. How the

304 barley UDP-glucosyltransferase performs in wheat under its native barley promoter

305 has not yet been demonstrated and hence the increase in resistance attributed to the

306 barley UGT-glucosyltransferase in wheat may be due to overexpression. The barley

307 UDP-glucosyltransferase UGT13248 is encoded by gene HORVU5Hr1G047150

308 which is present near the centromere on chromosome $5 \mathrm{H}$ (Ensembl Plants). If the

309 breakpoints in the wheat - barley 5HS and 5HL ditelosomic addition lines are not

310 centromeric, this may explain the findings related to the high level of resistance

311 conferred by addition of both $5 \mathrm{HS}$ and $5 \mathrm{HL}$. To confirm this, we designed primers

312 specific to the barley copy of the UDP-glucosyltransferase and will not amplify from

313 the orthologous wheat copies in the wheat-barley additions. This assay confirmed

314 that the UDP-glucosyltransferase was isolated to the $5 \mathrm{HL}$ addition line and was

315 absent in the 5HS addition line. Hence, it is likely that an independent source of FHB

316 resistance is present on $5 \mathrm{HS}$.

317 In this study, we also found that addition of the barley chromosome $7 \mathrm{H}(7 \mathrm{H}$ add $)$ or

318 the short arm of chromosome $1 \mathrm{H}$ (1HS add), as well as the translocation of $1 \mathrm{H}$ to $2 \mathrm{D}$

319 (2D-1H trans), significantly increased Type II FHB resistance in the winter wheat

320 variety Mv9kr1. Despite the enhanced FHB resistance from the addition of $7 \mathrm{H}$ to

$321 \mathrm{Mv9kr1}$, the addition of neither 7HS nor 7HL had an effect in the Asian spring wheat

322 cultivar Chinese Spring. No 1H addition lines were available in the Chinese Spring-

323 Betzes addition set, so this could not be compared between populations. These

324 findings suggest that barley contains genes conferring Type II resistance that are

325 lacking in one or both wheat varieties. The addition of barley chromosomes $5 \mathrm{H}$ and 
326 perhaps $1 \mathrm{H}$ and $7 \mathrm{H}$ are likely to offer the best opportunity of enhancing $\mathrm{FHB}$

327 resistance, when considering the use of wheat-barley introgressions.

328 We confirmed the presence of a possible Type II susceptibility factor on the short

329 arm of 4D in three independent experiments. The loss of 4DS (line DT(4DL))

330 resulted in a high level of FHB resistance, whilst the loss of 4DL (line DT(4DS))

331 resulted in little change compared to euploid Chinese Spring. Ma et al. (2006)

332 phenotyped Chinese Spring ditelosomic lines for FHB susceptibility and they also

333 reported an increase in FHB resistance in the line missing 4DS. Together, these

334 studies strongly suggest the presence of a susceptibility factor in both winter

335 (Mv9kr1) and spring (Chinese Spring) wheat genetic backgrounds. We applied

336 purified DON to the 4D ditelosomic lines to test whether or not the susceptibility

337 factor is being influenced by DON. However, the loss of 4DS resulted in higher

338 susceptibility to DON, assessed both by scoring DON induced bleaching and by

339 comparing grain weights. This would indicate that there is an independent resistance

340 factor to DON present on 4DS and that the susceptibility factor is increasing

341 susceptibility to the fungus or another virulence factor.

342 Endo and Gill (1996) developed a set of terminal deletion lines in Chinese Spring.

343 The lines have deletions from the ends of each chromosome arm, varying in size.

344 These stocks are a valuable resource for physically mapping genes to a defined

345 interval of a chromosome arm. The lines were characterised using C-banding and

346 the deletion size reported as a fraction length (FL) value; effectively the proportion of

347 the chromosome arm estimated to have been retained. C-banding is unlikely to be

348 capable of reliably detecting more complex deletions, such as interstitial deletions or

349 chromosome substitutions. Since their development, the Chinese Spring terminal

350 deletion stocks have not been more precisely characterised using more recent

351 advancements in genotyping. We have genotyped four lines containing terminal

352 deletions of 4DS, using a total of 37 novel homoeologue nonspecific markers

353 spanning the chromosome arm. These markers take advantage of the hexaploid

354 nature of wheat to create a robust genotyping assay for the detection of deletions on

355 4DS, and its homoeologous regions on 4BS and 4AL. A similar assay was used by

356 Chia et al. (2017) to verify deletions across homoeologous regions but this study

357 expands on this technique, using a much higher density of markers to characterise

358 deletion size. Homoeologous genes are simultaneously amplified with a single pair of 
359 primers but are distinguishable due to differences in the size of PCR products 360 corresponding to the A, B and D genome copies. The signal from the retained 361 homoeologues act as internal controls for a deletion in any homoeologue; in this 362 case, the 4D copy. This technique verified that all four Chinese Spring 4DS terminal 363 deletion lines were indeed true terminal deletions and the size of the deletions were 364 consistent with the FL values calculated by Endo and Gill (1996). For the lines 365 del4DS-2, del4DS-4 and del4DS-3, the physical position of the deletion endpoint has 366 been restricted to a small interval. For both del4DS-2 and del4DS-4, this interval is 367 smaller than $1 \mathrm{Mbp}$. The interval containing the deletion endpoint in del4DS-3 has 368 been refined to approximately 2.3 Mbp. The breakpoint in the largest deletion, 369 del4DS-1, was less precisely characterised and the deletion breakpoint was isolated 370 to a $29.8 \mathrm{Mbp}$ interval. For the purposes of this study, it was not necessary to more 371 precisely characterise the deletion in del4DS-1, because the FHB susceptibility 372 factor appears to be situated between the deletion breakpoints in lines del4DS-4 and 373 del4DS-3.

374 We performed FHB disease experiments on the four Chinese Spring 4DS terminal 375 deletion lines that we genotyped. This clearly demonstrated that the lines with the 376 two smaller deletions, del4DS-2 and del4DS-4, retained the susceptibility factor and 377 showed a similar phenotype to euploid Chinese Spring. In contrast the lines del4DS3783 and del4DS-1, containing the larger deletions, showed significantly improved FHB 379 resistance and hence the susceptibility factor has presumably been lost. As the 380 susceptibility factor was present in del4DS-4 but was lost in del4DS-3, it must be 381 situated between the deletion breakpoints of these two lines, restricting the 382 susceptibility factor to a $31.7 \mathrm{Mbp}$ interval containing 274 high confidence genes 383 (IWGSC RefSeq v1.1). The positive effect of the deletion of the susceptibility factor 384 appears to be restricted to $4 \mathrm{D}$ and hence it is likely the gene responsible is $4 \mathrm{D}$ 385 specific and does not possess homoeologues. BLAST searches of each 4D gene in 386 the interval identified 20 genes that appear to lack homoeologues and hence are 4D387 specific. Alternatively, the 4D homoeologue may be preferentially expressed 388 compared to the $4 \mathrm{~A}$ and $4 \mathrm{~B}$ copies. It is also possible that the improved $\mathrm{FHB}$ 389 resistance is the consequence of altered dosage of the 4D susceptibility factor and 390 its homoeologues. The disrupted balance of a physiological process exploited by the 391 fungus is also likely to result in altered disease susceptibility. 
392 A population possessing smaller deletions is required to further refine the position of 393 the FHB susceptibility factor. We intend to utilise a gamma-irradiated population of 394 the UK spring wheat variety Paragon (Shaw et al., 2013; Wheat Genetic 395 Improvement Network, 2019) to improve the resolution for the physical mapping of 396 the FHB susceptibility factor.

397 It may be considered surprising that an FHB susceptibility factor with such a powerful 398 effect has not been detected before now. However, we hypothesise that the FHB 399 susceptibility factor is highly conserved among wheat cultivars. The susceptibility 400 factor exists both in the Hungarian winter wheat cultivar Martonvasari 9 and in the 401 Asian spring wheat variety Chinese Spring. Preliminary experiments of gamma 402 irradiated Paragon lines, containing a deletion of the entire 31.7 Mbp FHB 403 susceptibility interval, indicated this line possesses potent resistance and hence 404 confirms that the susceptibility factor is also present in the UK spring cultivar 405 Paragon (data not shown). If there was sufficient allelic variation at the locus, the 406 effect of the susceptibility factor is likely to have been detected as an FHB QTL in 407 existing mapping populations. In the absence of such reports, we predict that the 408 FHB susceptibility factor is fixed in both spring and winter wheats.

409 Genetic resistance to fungal diseases is critical to the protection of food crops such 410 as wheat. The search and incorporation of resistance factors is common practice in 411 crop plant breeding. However, identifying novel sources of resistance to FHB is 412 challenging and time consuming. FHB resistance is quantitative, highly polygenic, 413 and often environmentally labile. Few large effect FHB QTL have been identified. 414 Attempts to clone the gene underlying the best known source of FHB resistance, the 415 Fhb1 QTL, have been inconsistent and controversial (Ma et al., 2017; Rawat et al., 416 2017; Steiner et al., 2017; Su et al., 2017). Rawat et al. (2016) reported that they had 417 cloned a pore-forming toxin-like (PFT) gene underlying the Fhb1 QTL. However, Jia 418 et al. (2018) disputed the findings of Rawat et al. (2016). Su et al. (2018) identified 419 that the presence of a deletion at the 5 ' end of a histidine-rich calcium-binding 420 protein within the Fhb1 locus was sufficient in identifying varieties carrying Fhb1. Su 421 et al. (2019) have since reported that Fhb1 possesses enhanced resistance due to 422 the loss-of-function of the histidine-rich calcium-binding protein and the wild type 423 allele is hence functioning as a susceptibility factor. Li et al. (2019) also identified 424 that mutation of the histidine-rich calcium-binding protein as the gene responsible for 
425 Fhb1 resistance. However, in conflict with the findings of Su et al. (2019), their data 426 suggests that this is due to a gain-of-function resulting from an different start codon

427 positioned upstream to the original (Li et al., 2019). Our data on the 3HS-3BL centric 428 fusion line does not suggest that 3BS contains a susceptibility factor, as the line was 429 either wild type-like or more highly susceptible to the spread of FHB. Furthermore, 430 Ma et al. (2006) reported that the Chinese Spring ditelosomic line missing 3BS 431 (DT(3BL)) was more susceptible to $\mathrm{FHB}$, which is not compatible with the hypothesis 432 that FHB resistance from Fhb1 being a loss-of-function susceptibility factor. It 433 remains possible that more than one gene is responsible for FHB resistance 434 conferred by Fhb1. Furthermore, it has proven difficult to utilise Fhb1 in elite varieties 435 in high yielding European environments with few varieties released containing the 436 resistance. This suggests a linkage drag from the resistance or a pleiotropic effect 437 and demonstrates a need for novel methods of conferring resistance, such as 438 eliminating susceptibility factors.

439 Despite this, there has been relatively little research into susceptibility factors in 440 wheat and other cereals and how they may be used in plant breeding. The barley 441 mildew resistance locus $O(M / O)$ is one of the earliest and best characterised 442 examples of how disruption of a susceptibility factor could be exploited to improve 443 disease resistance; in this case, to powdery mildew caused by the biotrophic fungus 444 Blumeria graminis f. sp. hordei (Jorgensen, 1992). Induced and natural mutation of 445 the Mlo locus result is a recessive, race nonspecific and durable resistance which 446 has been widely deployed in European spring barley varieties (Jorgensen, 1992;

447 Lyngkjaer and Carver, 2000; McGrann et al., 2014). Mlo-based resistance has since 448 been demonstrated in a number of other species affected by powdery mildew, 449 reviewed by Kusch and Panstruga (2017). The deployment of $m l o$ in wheat is more 450 challenging due to its allohexaploid nature (Acevedo-Garcia et al., 2017). However, 451 TALENs and CRISPR Cas9- derived gene knockouts (Wang et al., 2014) and Mlo 452 TILLING mutants (Acevedo-Garcia et al., 2017) have been used to demonstrate that 453 mutation of all wheat copies strongly enhances resistance to wheat powdery mildew.

454 R genes, usually nucleotide binding site- leucine rich repeat (NBS-LRR) genes, are 455 typically used by plants to detect and respond to attack by biotrophic fungi. However, 456 necrotrophic pathogens have evolved methods of exploiting such plant defences to 457 aid infection. Parastagonospora nodorum and Pyrenophora tritici-repentis are 
458 necrotrophic pathogens of wheat that utilise this strategy. Susceptibility to these

459 diseases operates in an inverse gene-for-gene interaction, in which a fungal

460 necrotrophic effector is detected by a corresponding host sensitivity gene product

461 (usually an NBS-LRR), triggering a hypersensitive response that results in necrosis

462 that benefits the fungus (Faris et al., 2010). If either necrotrophic effector or host

463 sensitivity gene is absent, the interaction is impossible and host resistance is

464 maintained. There have been few reports of how NBS-LRRs are involved in

465 interactions with Fusarium spp. However, Zhang et al. (2019) found that the

466 expression of an LRR gene appeared to increase susceptibility to F. graminearum in

467 soybean (Glycine max).

468 Fusarium graminearum leads a hemibiotrophic lifestyle whereby the hyphal front

469 remains surrounded by living tissue but cell death is triggered soon after colonisation

470 (Brown et al., 2010). Phytohormones play important roles in defence and there is

471 considerable evidence indicating that $F$. graminearum modifies phytohormone

472 expression for its own benefit. Disruption of ethylene signalling in wheat (Chen et al.,

473 2009) and brassinosteroid signalling in barley and Brachypodium distachyon

474 (Goddard et al., 2014) results in enhanced resistance to FHB infection, suggesting

475 that the fungus is exploiting phytohormone signalling in order to aid infection.

476 Expression of 9-lipogenases are also manipulated by F. graminearum in both bread

477 wheat and Arabidopsis thaliana and are hence operating as susceptibility factors

478 (Nalam et al., 2015).

479 In this study, we provide compelling evidence for the presence of an FHB

480 susceptibility factor on the short arm of chromosome 4D. We have demonstrated that

481 the removal of the susceptibility factor is sufficient to significantly improve Type II

482 FHB resistance and have refined its position to a 31.7 Mbp interval containing 274

483 high confidence genes. We have designed markers that can reliably detect deletions

484 on 4DS. A subset of these markers covering the susceptibility interval will be utilised

485 in further studies to identify lines containing relatively smaller deletions across the

486 FHB susceptibility interval in a gamma irradiated Paragon population. This will

487 reduce the number of gene candidates for the FHB susceptibility and may lead to the

488 identification of the causal gene. 


\section{Acknowledgements}

490 The authors would like to thank BBSRC (grant number: BB/M016919/1) and RAGT

491 Seeds for supporting the PhD studentship of $\mathrm{BH}$. This work was supported by

492 BBSRC and the John Innes Foundation. 


\section{References}

Acevedo-Garcia J, Spencer D, Thieron H, Reinstadler A, Hammond-Kosack K, Phillips AL, Panstruga R. 2017. mlo-based powdery mildew resistance in hexaploid bread wheat generated by a non-transgenic TILLING approach. Plant Biotechnology Journal 15, 367-378.

Altpeter F, Posselt UK. 1994. Production of high quantities of 3acetyldeoxynivalenol and deoxynivalenol. Applied Microbiology and Biotechnology 41, 384-387.

Bai GH, Desjardins AE, Plattner RD. 2002. Deoxynivalenol-nonproducing Fusarium graminearum causes initial infection, but does not cause disease spread in wheat spikes. Mycopathologia 153, 91-98.

Boutigny AL, Richard-Forget F, Barreau C. 2008. Natural mechanisms for cereal resistance to the accumulation of Fusarium trichothecenes. European Journal of Plant Pathology 121, 411-423.

Brown NA, Urban M, Van De Meene AML, Hammond-Kosack KE. 2010. The infection biology of Fusarium graminearum: Defining the pathways of spikelet to spikelet colonisation in wheat ears. Fungal Biology 114, 555-571.

Buerstmayr H, Ban T, Anderson JA. 2009. QTL mapping and marker-assisted selection for Fusarium head blight resistance in wheat: a review. Plant Breeding 128, 1-26.

Buerstmayr M, Steiner B, Buerstmayr H. 2019. Breeding for Fusarium head blight resistance in wheat-Progress and challenges. Plant Breeding, 26.

Chen X, Steed A, Travella S, Keller B, Nicholson P. 2009. Fusarium graminearum exploits ethylene signalling to colonize dicotyledonous and monocotyledonous plants. New Phytologist 182, 975-983.

Chia T, Adamski NM, Saccomanno B, Greenland A, Nash A, Uauy C, Trafford K. 2017. Transfer of a starch phenotype from wild wheat to bread wheat by deletion of a locus controlling B-type starch granule content. Journal of Experimental Botany 68, 5497-5509.

Endo TR, Gill BS. 1996. The deletion stocks of common wheat. Journal of Heredity 87, 295-307.

Faris JD, Zhang ZC, Lu HJ, Lu SW, Reddy L, Cloutier S, Fellers JP, Meinhardt SW, Rasmussen JB, Xu SS, Oliver RP, Simons KJ, Friesen TL. 2010. A unique wheat disease resistance-like gene governs effector-triggered susceptibility to necrotrophic pathogens. Proceedings of the National Academy of Sciences of the United States of America 107, 13544-13549.

Garvin DF, Porter H, Blankenheim ZJ, Chao SM, Dill-Macky R. 2015. A spontaneous segmental deletion from chromosome arm 3DL enhances Fusarium head blight resistance in wheat. Genome 58, 479-488.

Goddard R, Peraldi A, Ridout C, Nicholson P. 2014. Enhanced Disease Resistance Caused by BRI1 Mutation Is Conserved Between Brachypodium distachyon and Barley (Hordeum vulgare). Molecular Plant-Microbe Interactions 27, 1095-1106.

Gosman N, Chandler E, Thomsett M, Draeger R, Nicholson P. 2005. Analysis of the relationship between parameters of resistance to Fusarium head blight and in vitro tolerance to deoxynivalenol of the winter wheat cultivar WEK0609 (R).

European Journal of Plant Pathology 111, 57-66.

Gunupuru LR, Perochon A, Doohan FM. 2017. Deoxynivalenol resistance as a component of FHB resistance. Tropical Plant Pathology 42, 175-183. 
Hassan YI, He JW, Perilla N, Tang KJ, Karlovsky P, Zhou T. 2017. The enzymatic epimerization of deoxynivalenol by Devosia mutans proceeds through the formation of 3-keto-DON intermediate. Scientific Reports 7, 11.

He WJ, Zhang LM, Yi SY, Tang XL, Yuan QS, Guo MW, Wu AB, Qu B, Li HP, Liao YC. 2017. An aldo-keto reductase is responsible for Fusarium toxin-degrading activity in a soil Sphingomonas strain. Scientific Reports 7, 13.

Islam AKMR, Shepherd KW, Sparrow DHB. 1981. Isolation and characterization of euplasmic wheat-barley chromosome addition lines. Heredity 46, 161-\&.

IWGSC. 2018. Shifting the limits in wheat research and breeding using a fully annotated reference genome. Science 361.

Jia HY, Zhou JY, Xue SL, Li GQ, Yan HS, Ran CF, Zhang YD, Shi JX, Jia L, Wang X, Luo J, Ma ZQ. 2018. A journey to understand wheat Fusarium head blight resistance in the Chinese wheat landrace Wangshuibai. Crop Journal 6, 48-59.

Jorgensen JH. 1992. Discovery, characterization and exploitation of Mlo powdery mildew resistance in barley. Euphytica 63, 141-152.

Kruppa K, Sepsi A, Szakacs E, Rodder MS, Molnar-Lang M. 2013.

Characterization of a 5HS-7DS.7DL wheat-barley translocation line and physical mapping of the 7D chromosome using SSR markers. Journal of Applied Genetics 54, 251-258.

Kusch S, Panstruga R. 2017. mlo-Based Resistance: An Apparently Universal "Weapon" to Defeat Powdery Mildew Disease. Molecular Plant-Microbe Interactions 30, 179-189.

Langevin F, Eudes F, Comeau A. 2004. Effect of trichothecenes produced by Fusarium graminearum during Fusarium head blight development in six cereal species. European Journal of Plant Pathology 110, 735-746.

Lemmens M, Scholz U, Berthiller F, Dall'Asta C, Koutnik A, Schuhmacher R, Adam G, Buerstmayr H, Mesterhazy A, Krska R, Ruckenbauer P. 2005. The ability to detoxify the mycotoxin deoxynivalenol colocalizes with a major quantitative trait locus for fusarium head blight resistance in wheat. Molecular Plant-Microbe Interactions 18, 1318-1324.

Li G, Zhou J, Jia H, Gao Z, Fan M, Luo Y, Zhao P, Xue S, Li N, Yuan Y, Ma S, Kong Z, Jia L, An X, Jiang G, Liu W, Cao W, Zhang R, Fan J, Xu X, Liu Y, Kong Q, Zheng S, Wang Y, Qin B, Cao S, Ding Y, Shi J, Yan H, Wang X, Ran C, Ma Z. 2019. Mutation of a histidine-rich calcium-binding-protein gene in wheat confers resistance to Fusarium head blight. Nature Genetics 51, 1106-1112.

Li X, Shin S, Heinen S, Dill-Macky R, Berthiller F, Nersesian N, Clemente T, McCormick S, Muehlbauer GJ. 2015. Transgenic Wheat Expressing a Barley UDPGlucosyltransferase Detoxifies Deoxynivalenol and Provides High Levels of Resistance to Fusarium graminearum. Molecular Plant-Microbe Interactions 28, 1237-1246.

Lucyshyn D, Busch BL, Abolmaali S, Steiner B, Chandler E, Sanjarian F, Mousavi A, Nicholson P, Buerstmayr H, Adam G. 2007. Cloning and characterization of the ribosomal protein L3 (RPL3) gene family from Triticum aestivum. Molecular Genetics and Genomics 277, 507-517.

Lyngkjaer MF, Carver TLW. 2000. Conditioning of cellular defence responses to powdery mildew in cereal leaves by prior attack. Molecular Plant Pathology 1, 41-49. Ma HX, Bai GH, Gill BS, Hart LP. 2006. Deletion of a chromosome arm altered wheat resistance to Fusarium head blight and deoxynivalenol accumulation in Chinese Spring. Plant Disease 90, 1545-1549. 
Ma Z, Li G, Zhou J, Jia H, Xue S, Li N, Gao Z, Fan M, Zhang R, Ding Y, Kong Z, Yuan Y, Ma S, Jia L, Ran C. 2017. Map-based cloning of Fhb1 revealed unique mutation of a well-conserved gene resulting in resistance to wheat Fusarium head blight In: Buerstmayr H, Lang-Mladek C, Steiner B, Michel S, Buerstmayr M, Lemmens M, Vollmann J, Grausgruber H, eds. 13th International Wheat Genetics Symposium. Tulln, Austria: BOKU - University of Natural Resources and Life Sciences, Vienna, 68.

Maier FJ, Miedaner T, Hadeler B, Felk A, Salomon S, Lemmens M, Kassner H, Schafer W. 2006. Involvement of trichothecenes in fusarioses of wheat, barley and maize evaluated by gene disruption of the trichodiene synthase (Tri5) gene in three field isolates of different chemotype and virulence. Molecular Plant Pathology 7, 449461.

McGrann GRD, Stavrinides A, Russell J, Corbitt MM, Booth A, Chartrain L, Thomas WTB, Brown JKM. 2014. A trade off between mlo resistance to powdery mildew and increased susceptibility of barley to a newly important disease, Ramularia leaf spot. Journal of Experimental Botany 65, 1025-1037.

Mitterbauer R, Poppenberger B, Raditschnig A, Lucyshyn D, Lemmens M, GlossI J, Adam G. 2004. Toxin-dependent utilization of engineered ribosomal protein L3 limits trichothecene resistance in transgenic plants. Plant Biotechnology Journal 2, 329-340.

Molnar I, Linc G, Dulai S, Nagy ED, Molnar-Lang M. 2007. Ability of chromosome $4 \mathrm{H}$ to compensate for 4D in response to drought stress in a newly developed and identified wheat-barley 4H(4D) disomic substitution line. Plant Breeding 126, 369374.

MolnarLang M, Linc G, Sutka J. 1996. Transfer of the recessive crossability allele $\mathrm{kr} 1$ from Chinese Spring into the winter wheat variety Martonvasari 9. Euphytica 90, 301-305.

Nagy ED, Molnar-Lang M, Linc G, Lang L. 2002. Identification of wheat-barley translocations by sequential GISH and two-colour FISH in combination with the use of genetically mapped barley SSR markers. Genome 45, 1238-1247.

Nalam VJ, Alam S, Keereetaweep J, Venables B, Burdan D, Lee H, Trick HN, Sarowar S, Makandar R, Shah J. 2015. Facilitation of Fusarium graminearum Infection by 9-Lipoxygenases in Arabidopsis and Wheat. Molecular Plant-Microbe Interactions 28, 1142-1152.

Pallotta M, Warner P, Fox RL, Kuchel H, Jefferies SJ, Langridge P. 2003. Marker assisted wheat breeding in the southern region of Australia. In: Pogna N, Mclntosh $\mathrm{R}$, eds. Proceedings of the 10th international wheat genetics symposium Vol. 2. Paestum, Italy: Istituto Sperimentale per per la Cerealicoltura 789-791.

Poppenberger B, Berthiller F, Lucyshyn D, Sieberer T, Schuhmacher R, Krska R, Kuchler K, GlossI J, Luschnig C, Adam G. 2003. Detoxification of the Fusarium mycotoxin deoxynivalenol by a UDP-glucosyltransferase from Arabidopsis thaliana. Journal of Biological Chemistry 278, 47905-47914.

Rawat N, Pumphrey MO, Liu S, Zhang X, Tiwari VK, Ando K, Trick HN, Bockus WW, Akhunov E, Anderson JA, Gill BS. 2016. Wheat Fhb1 encodes a chimeric lectin with agglutinin domains and a pore-forming toxin-like domain conferring resistance to Fusarium head blight. Nat Genet advance online publication. Rawat N, Pumphrey MO, Liu S, Zhang X, Tiwari VK, Trick HN, Akhunov E, Anderson JA, Gill BS. 2017. Pore-forming toxin-like gene provides resistance against Fusarium head blight in wheat In: Buerstmayr $\mathrm{H}$, Lang-Mladek C, Steiner B, Michel S, Buerstmayr M, Lemmens M, Vollmann J, Grausgruber H, eds. 13th 
International Wheat Genetics Symposium. Tulln, Austria: BOKU - University of Natural Resources and Life Sciences, Vienna, 67.

Scherm B, Balmas V, Spanu F, Pani G, Delogu G, Pasquali M, Migheli Q. 2013. Fusarium culmorum: causal agent of foot and root rot and head blight on wheat. Molecular Plant Pathology 14, 323-341.

Schroeder HW, Christensen JJ. 1963. Factors affecting resistance of wheat to scab caused by Gibberella zeae. Phytopathology 53, 831-838.

Schuelke M. 2000. An economic method for the fluorescent labeling of PCR fragments. Nature Biotechnology 18, 233-234.

Schweiger W, Boddu J, Shin S, Poppenberger B, Berthiller F, Lemmens M, Muehlbauer GJ, Adam G. 2010. Validation of a Candidate DeoxynivalenolInactivating UDP-Glucosyltransferase from Barley by Heterologous Expression in Yeast. Molecular Plant-Microbe Interactions 23, 977-986.

Shaw LM, Turner AS, Herry L, Griffiths S, Laurie DA. 2013. Mutant Alleles of Photoperiod-1 in Wheat (Triticum aestivum L.) That Confer a Late Flowering Phenotype in Long Days. Plos One 8, 11.

Steiner B, Zimmerl S, Polzer R, Mühl S, Lemmens M, Adam G, Till B, Schweiger W, Buerstmayr H. 2017. Functional identification of the wheat gene enhancing mycotoxin detoxification of the major Fusarium resistance QTL Fhb1. In: Buerstmayr $\mathrm{H}$, Lang-Mladek C, Steiner B, Michel S, Buerstmayr M, Lemmens M, Vollmann J, Grausgruber H, eds. 13th International Wheat Genetics Symposium. Tulln, Austria: BOKU - University of Natural Resources and Life Sciences, Vienna, 70.

Su Z, Bernardo A, Li C, Lu P, Cai S, Bai G. 2017. TaHRC is the key gene underlying Fhb1 resistance to Fusarium head blight in wheat In: Buerstmayr $\mathrm{H}$, Lang-Mladek C, Steiner B, Michel S, Buerstmayr M, Lemmens M, Vollmann J, Grausgruber H, eds. 13th International Wheat Genetics Symposium. Tulln, Austria: BOKU - University of Natural Resources and Life Sciences, Vienna, 69.

Su Z, Bernardo A, Tian B, Chen H, Wang S, Ma H, Cai S, Liu D, Zhang D, Li T, Trick H, St. Amand P, Yu J, Zhang Z, Bai G. 2019. A deletion mutation in TaHRC confers Fhb1 resistance to Fusarium head blight in wheat. Nature Genetics 51, 1099-1105.

Su ZQ, Jin SJ, Zhang DD, Bai GH. 2018. Development and validation of diagnostic markers for Fhb1 region, a major QTL for Fusarium head blight resistance in wheat. Theoretical and Applied Genetics 131, 2371-2380.

Szakacs E, Molnar-Lang M. 2007. Development and molecular cytogenetic identification of new winter wheat-winter barley ('Martonvasari 9 kr1'-'Igri') disomic addition lines. Genome 50, 43-50.

Szakacs E, Molnar-Lang M. 2010. Identification of new winter wheat - winter barley addition lines (6HS and $7 \mathrm{H}$ ) using fluorescence in situ hybridization and the stability of the whole 'Martonvasari 9 kr1' - 'Igri' addition set. Genome 53, 35-44.

Wang YP, Cheng X, Shan QW, Zhang Y, Liu JX, Gao CX, Qiu JL. 2014. Simultaneous editing of three homoeoalleles in hexaploid bread wheat confers heritable resistance to powdery mildew. Nature Biotechnology 32, 947-951.

Wheat Genetic Improvement Network. 2019. Wheat Genetic Improvement Network. Vol. 2019: Rothamsted Research.

Xing LP, Gao L, Chen QG, Pei HY, Di ZC, Xiao J, Wang HY, Ma LL, Chen PD, Cao AZ, Wang XE. 2018. Over-expressing a UDP-glucosyltransferase gene (TaUGT (3) ) enhances Fusarium Head Blight resistance of wheat. Plant Growth Regulation 84, 561-571. 
Zhang CJ, Zhao X, Qu YF, Teng WL, Qiu LJ, Zheng HK, Wang ZH, Han YP, Li WB. 2019. Loci and candidate genes in soybean that confer resistance to Fusarium graminearum. Theoretical and Applied Genetics 132, 431-441. 


\section{Tables}

Table 1 Wheat-barley addition, substitution, translocation and centric fusion lines used in FHB experiments.

The primary wheat parent was Martonvasari 9 kr1 (Mv9kr1) for all lines and the barley donor parents were Igri or Betzes. Associated references contain detailed descriptions of line generation and composition.

\begin{tabular}{|l|l|l|}
\hline $\begin{array}{l}\text { Line } \\
\text { abbreviation }\end{array}$ & Description & Reference \\
\hline Mv9kr1 & Martonvasari9 kr1 & MolnarLang et al. (1996) \\
\hline 1HS add & Mv9kr1-Igri 1HS disomic addition & Szakacs and Molnar-Lang (2007) \\
\hline 2H add & Mv9kr1-Igri 2H disomic addition & Szakacs and Molnar-Lang (2007) \\
\hline 3H add & Mv9kr1-Igri 3H disomic addition & Szakacs and Molnar-Lang (2007) \\
\hline 4H add & Mv9kr1-Igri 4H disomic addition & Szakacs and Molnar-Lang (2007) \\
\hline 6HS add & Mv9kr1-Igri 6HS disomic addition & Szakacs and Molnar-Lang (2010) \\
\hline 7H add & Mv9kr1-Igri 7H disomic addition & Szakacs and Molnar-Lang (2010) \\
\hline 2D-1H trans & 2DS.2DL-1HS translocation & Nagy et al. (2002) \\
\hline 3HS.3BL centric & 3HS.3BL centric fusion & Nagy et al. (2002) \\
\hline 4H(4D) sub & 4H(4D) wheat-barley substitution & Molnar et al. (2007) \\
\hline 6B-4H trans & 6BS.6BL-4HL translocation & Nagy et al. (2002) \\
\hline 7D-5H trans & 5HS-7DS.7DL wheat-barley translocation & Kruppa et al. (2013) \\
\hline
\end{tabular}


Table 2 Homoeologue nonspecific markers used to genotype four Chinese Spring 4DS terminal deletion lines.

Primer sequences, fragment sizes (corresponding to the 4A, 4B and 4D homoeologous gene targets) and the 4D gene target of markers used to characterise the deletion sizes present in four Chinese Spring 4DS terminal deletion lines. The lowercase sequence in the forward primer indicates the M13 tail. All markers amplified at $58^{\circ} \mathrm{C}$ annealing temperature.

\begin{tabular}{|c|c|c|c|c|}
\hline Marker & Forward primer & Reverse primer & Fragment A; B; D (bp) & 4D gene target \\
\hline BH0001 & tgtaaaacgacggccagtTCCTCCAATAAGAAGGTATGTC & TGGCACTGCCCTTATAGCAA & $356 ; 330 ; 228$ & TraesCS4D02G001400 \\
\hline $\mathrm{BHOOO2}$ & tgtaaaacgacggccagtTGTCGTTGTTCCAGTTAAAG & TCAGGCGCATCAGACATTTG & $205 ; 172 ; 163$ & TraesCS4D02G009200 \\
\hline BH0013 & tgtaaaacgacggccagtGGGGAATTGTCCAAAGCGT & TGCAAGAGATGTTGGGATTTT & $211 ; 155 ; 207$ & TraesCS4D02G014500 \\
\hline BHOOO3 & tgtaaaacgacggccagtCTCCACTTTATCATTTGAAGACA & ACAAAACCTTTCACATGGCC & $452 ; 264 ; 491$ & TraesCS4D02G017300 \\
\hline BHOOO4.2 & tgtaaaacgacggccagtGTGTTCCCATTGTCGCCG & TAGTCCGCCTCCTTGCTCCT & $168 ; 152 ; 194$ & TraesCS4D02G035700 \\
\hline BH0025.2 & tgtaaaacgacggccagtACAATCCCGAGGTTGCCAGA & CGAAGAGGAGGGCATACATA & $275 ; 359 ; 378$ & TraesCS4D02G039400 \\
\hline BH0005.2 & tgtaaaacgacggccagtTGGTGCTTCATTATCCTTCTGAT & TGGTGTCCAGAGTAAACTCGATA & $443 ; 448 ; 319$ & TraesCS4D02G040700 \\
\hline BH0O2O & tgtaaaacgacggccagtCGACCTCCTCTCAGCTTTTAG & ATGAGGATACACGGTGCTGC & $304 ; 193 ; 220$ & TraesCS4D02G045500 \\
\hline $\mathrm{BH} 0029$ & tgtaaaacgacggccagtGAGCAGATCTTCAACGTACG & ATCACAAAGGGATGGACCTG & $183 ; 196 ; 159$ & TraesCS4D02G050300 \\
\hline BH0O24 & tgtaaaacgacggccagtAAAGTAAAATCCTCTTCCCTGAG & GCTAAACTTGCTGTCAGACAAG & $274 ; 298 ; 389$ & TraesCS4D02G051400 \\
\hline BH0006.2 & tgtaaaacgacggccagtGGCCAAGGTGCGTAATCCA & CGCGAGCTGAACACAAGC & $265 ; 121 ; 313$ & TraesCS4D02G052300 \\
\hline $\mathrm{BH} 0022$ & tgtaaaacgacggccagtAGTATTAGGCAATGTGTTCCACT & TGAGAAGGTTCCAAGAACCAAC & $288 ; 459 ; 260$ & TraesCS4D02G057100 \\
\hline BH0O21 & tgtaaaacgacggccagtTCATTCAACATGCAGATCTAGGC & GACAAACTTCAATGGCATAAGC & $123 ; 155 ; 130$ & TraesCS4D02G065300 \\
\hline BH0014 & tgtaaaacgacggccagtCCATTGCATTCСTTCACTTGT & CGTCGTCCCATACTTCACAAA & $110 ; 113 ; 107$ & TraesCS4D02G066900 \\
\hline $\mathrm{BH} 0026$ & tgtaaacgacggccagtCGATACACCAGTTAATTGAAATATG & CTAGGAGTTCCTTCATGGACATT & $289 ; 471 ; 318$ & TraesCS4D02G073200 \\
\hline BH0015.2 & tgtaaaacgacggccagtCACAACTTGTGCAGGTATAACC & GGAAAGTCAAGACAGGCACAA & $198 ; 346 ; 426$ & TraesCS4D02G074200 \\
\hline BHOOO8 & tgtaaaacgacggccagtGTATCGACGAAGCCGCAGTT & TTCCGGAGCGTCCTACGACAA & $309 ; 190 ; 199$ & TraesCS4D02G074500 \\
\hline $\mathrm{BH} 0040$ & tgtaaaacgacggccagtGCGCAGTGAGACAAAACTC & AAGTAGAAGAGCAGCGCCAT & $442 ; 448 ; 451$ & TraesCS4D02G075300 \\
\hline BH0O41 & tgtaaaacgacggccagtAACAAATCCATGTGACCCC & CTACAAGGACGCGTGGTTAT & $299 ; 338 ; 302$ & TraesCS4D02G076000 \\
\hline $\mathrm{BH} 0042$ & tgtaaacgacggccagtCGGACAACATTTCAGGATTTC & ACCGGAACAAGGCTGCAC & $379 ; 135 ; 125$ & TraesCS4D02G077600 \\
\hline BH0O27.3 & tgtaaaacgacggccagtGGTAACATTCCTTTGGTATACTCGG & TGTGCTAAGATCTACAACATC & $303 ; 350 ; 266$ & TraesCS4D02G078900 \\
\hline $\mathrm{BH} 0032$ & tgtaaaacgacggccagtTTGTGGCCTGCTTACATTGC & TGATCTGCAGGTGTTGGC & $317 ; 305 ; 300$ & TraesCS4D02G079900 \\
\hline $\mathrm{BH} 0033$ & tgtaaaacgacggccagtTGCCCGTGTTTTATGCACTG & GGTAAGTAAAATGGGAAGAAAGC & $201 ; 167 ; 185$ & TraesCS4D02G081000 \\
\hline BH0O34 & tgtaaaacgacggccagtCTGCCGTATCTCCAACTC & ATGAGCGCCATCAGGAAC & $209 ; 297 ; 217$ & TraesCS4D02G082500 \\
\hline BH0O35 & tgtaaaacgacggccagtACGCGGACCCGAATTCAAA & TCCTTGGGCATAGAGGAAG & $190 ; 167 ; 162$ & TraesCS4D02G083100 \\
\hline $\mathrm{BH} 0036$ & tgtaaaacgacggccagtATGTTAGCCGTCCTTTGTTTC & TGGCTGACAGCTATACTTCTAGT & $246 ; 255 ; 223$ & TraesCS4D02G084000 \\
\hline BH0O37 & tgtaaaacgacggccagtGACGGACAATTCTTATGATTGTG & TATGTCCTGCCCCTTCTCCAT & $191 ; 187 ; 166$ & TraesCS4D02G085100 \\
\hline
\end{tabular}


Table 2 (continued) Homoeologue nonspecific markers used to genotype four Chinese Spring 4DS terminal deletion lines. Primer sequences, fragment sizes (corresponding to the 4A, 4B and 4D homoeologous gene targets) and the 4D gene target of markers used to characterise the deletion sizes present in four Chinese Spring 4DS terminal deletion lines. The lowercase sequence in the forward primer indicates the M13 tail. All markers amplified at $58^{\circ} \mathrm{C}$ annealing temperature.

\begin{tabular}{|c|c|c|c|c|}
\hline Marker & Forward primer & Reverse primer & $\begin{array}{l}\text { Fragment A; B; D } \\
\text { (bp) }\end{array}$ & 4D gene target \\
\hline $\mathrm{BH} 0038$ & tgtaaaacgacggccagtATCTGCGTCCAGGTGAGC & TCAGCTAAGACAACTGGCAC & $359 ; 341 ; 318$ & TraesCS4D02G085900 \\
\hline BH0009.3 & tgtaaaacgacggccagtTAGAGGGAGCAGGGATGACAT & TCTCCGTCTGGTTCATTCGT & $106 ; 103 ; 111$ & TraesCS4D02G087200 \\
\hline $\mathrm{BH} 0010.2$ & tgtaaaacgacggccagtACGTGGTCTTCAAATCTGGC & CTGCAATATAAGGTGGCAAATC & $189 ; 155 ; 159$ & TraesCS4D02G098400 \\
\hline $\mathrm{BH} 0017$ & tgtaaaacgacggccagtCAGATTGTACGAACATCTTCTGC & AGCAGAACAAAATCTCATGG & $252 ; 246 ; 263$ & TraesCS4D02G105100 \\
\hline $\mathrm{BH} 0018$ & tgtaaaacgacggccagtGTGAGCAGAGCACCCTCC & CTGCACCACCACAGAAAAGA & $226 ; 195 ; 214$ & TraesCS4D02G107300 \\
\hline $\mathrm{BH} 0011$ & tgtaaaacgacggccagtATGCTCGTCTTCATCGAGGTAA & ATGCATTGCAGACACATCAAG & $128 ; 160 ; 135$ & TraesCS4D02G114700 \\
\hline $\mathrm{BH} 0012.2$ & tgtaaaacgacggccagtGGTCCTTCATGAAGCTTGTTC & GGCAAATAAGAGAGTTGCATAGG & $275 ; 289 ; 280$ & TraesCS4D02G117800 \\
\hline $\mathrm{BH} 0030$ & tgtaaaacgacggccagtGGCAATGTGATCCTGCAGTTC & GCCCAAAGAAATAGCAAGGGAAA & $145 ; 174 ; 189$ & TraesCS4D02G126600 \\
\hline $\mathrm{BH} 0057$ & tgtaaaacgacggccagtGCACATCCTGCTGTACCA & CTCCTTGGGAATCTTAATGCA & $464 ; 356 ; 322$ & TraesCS4D02G147800 \\
\hline $\mathrm{BH} 0058$ & tgtaaaacgacggccagtCCATTTAGATTCATGGCGAT & AGGCATATTGCAAACCCAAC & $190 ; 315 ; 179$ & TraesCS4D02G149800 \\
\hline
\end{tabular}


Table 3 Flanking genes and markers of deletion breakpoints in four Chinese Spring 4DS terminal deletion lines.

The breakpoint interval is the size of the interval between two adjacent markers where the marker signal was retrieved, indicating the end of the deletion.

\begin{tabular}{|l|l|l|l|l|r|}
\hline Line & Left flank gene & $\begin{array}{l}\text { Left } \\
\text { flank } \\
\text { marker }\end{array}$ & Right flank gene & $\begin{array}{l}\text { Right } \\
\text { flank } \\
\text { marker }\end{array}$ & $\begin{array}{l}\text { Breakpoint } \\
\text { interval (Kb) }\end{array}$ \\
\hline del4DS-2 & TraesCS4D02G076000 & BH0041 & TraesCS4D02G077600 & BH0042 & 976 \\
\hline del4DS-4 & TraesCS4D02G079900 & BH0032 & TraesCS4D02G081000 & BH0033 & 949 \\
\hline del4DS-3 & TraesCS4D02G105100 & BH0017 & TraesCS4D02G107300 & BH0018 & 2313 \\
\hline del4DS-1 & TraesCS4D02G126600 & BH0030 & TraesCS4D02G147800 & BH0057 & 29776 \\
\hline
\end{tabular}




\section{Figure legends}

Figure $1 \mathrm{FHB}$ disease above inoculation point in wheat-barley addition, substitution, translocation and centric fusion lines from a) polytunnel experiment 1, including barley parents Igri and Betzes as controls, and b) polytunnel experiment 2. Predicted means were generated using a linear mixed model. Error bars are \pm standard error. * $p<0.05 ;{ }^{* *} p<0.01 ;{ }^{* * *} p<0.001$ compared to Mv9kr1.

Figure 2 FHB disease, as a percentage of total number of bleached spikelets, from data combined from $13 \mathrm{dpi}$ and $14 \mathrm{dpi}$. Predicted means were generated using a linear mixed model. Error bars are \pm standard error. * $p=0.05-0.01$ compared to Chinese Spring; ${ }^{* * *} p<0.001$ compared to Chinese Spring.

Figure $3 \mathrm{FHB}$ disease at $17 \mathrm{dpi}$ in euploid Chinese Spring and 4D ditelosomic lines DT(4DL) and DT(4DS), missing 4DS and 4DL, respectively. Diagrams of 4D are included above ditelosomic lines to illustrate their genetic state. Error bars are \pm standard error. ${ }^{* * *} p<0.001$ compared to Chinese Spring.

Figure $4 \mathrm{DON}$ application experiment to heads of Chinese Spring and ditelosomic lines $\mathrm{DT}(4 \mathrm{DL})$ and $\mathrm{DT}(4 \mathrm{DS})$, lacking $4 \mathrm{DS}$ and $4 \mathrm{DL}$, respectively. a) average DON bleaching scores at 7 days post application. Predicted means were generated using a linear mixed model. Error bars are \pm standard error. $p<0.001$ compared with Chinese Spring. b) ratio of DON treated/ untreated mean grain weight above the DON application point, or comparable point in untreated heads, dissected after the experiment. Ratios were calculated by subtracting the $\log 10$ mean grain weight of DON treated heads from untreated heads for each line, followed by back transformation to obtain a treated/untreated ratio for each line. Predicted means were generated using a linear mixed model. Error bars are \pm standard error. c) photograph showing three representative examples of untreated and DON treated grain taken from above the DON application point, or comparable point in untreated heads for each line.

Figure 5 Example outputs of five multiplexed markers BH0014 (left black), BH0030 (blue), BH0018 (red), BH0017 (green) and BH0026 (right black) in a) Chinese Spring; b) del4DS-2; c) del4DS-4; d) del4DS-1. The line del4DS-3 showed the same deletion pattern for the markers visible in the selected multiplex and was hence omitted. $X$ axis is fragment size (bp) and $Y$ axis is the strength of fluorescence (relative fluorescence units). Images were extracted as screenshots from Peak Scanner 2 software (Applied Biosystems).

Figure 6 FHB disease above the inoculation point at $13 \mathrm{dpi}$, following point inoculation of euploid Chinese Spring and four terminal deletion bins; del4DS-2, del4DS-4, del4DS-3 and del4DS-1. Error bars are \pm standard error. ${ }^{* * *} p<0.001$ compared to Chinese Spring.

Figure 7 Representative FHB disease symptoms in the Chinese Spring terminal deletion lines del4DS-4 and del4DS-3 at 16 dpi. 
Figure 8 Diagrams of 4DS in euploid Chinese Spring and four 4DS terminal deletion lines, as characterised by genotyping with 35 markers spanning 4DS. The spotted interval indicates the breakpoint interval; the distance between two markers where the 4D signal was retrieved. The bottom diagram indicates the interval on 4DS inferred to contain an FHB susceptibility factor (diagonal stripes), following point inoculation of the Chinese Spring terminal deletion lines. Values in bold indicate the physical position in Mbp. 


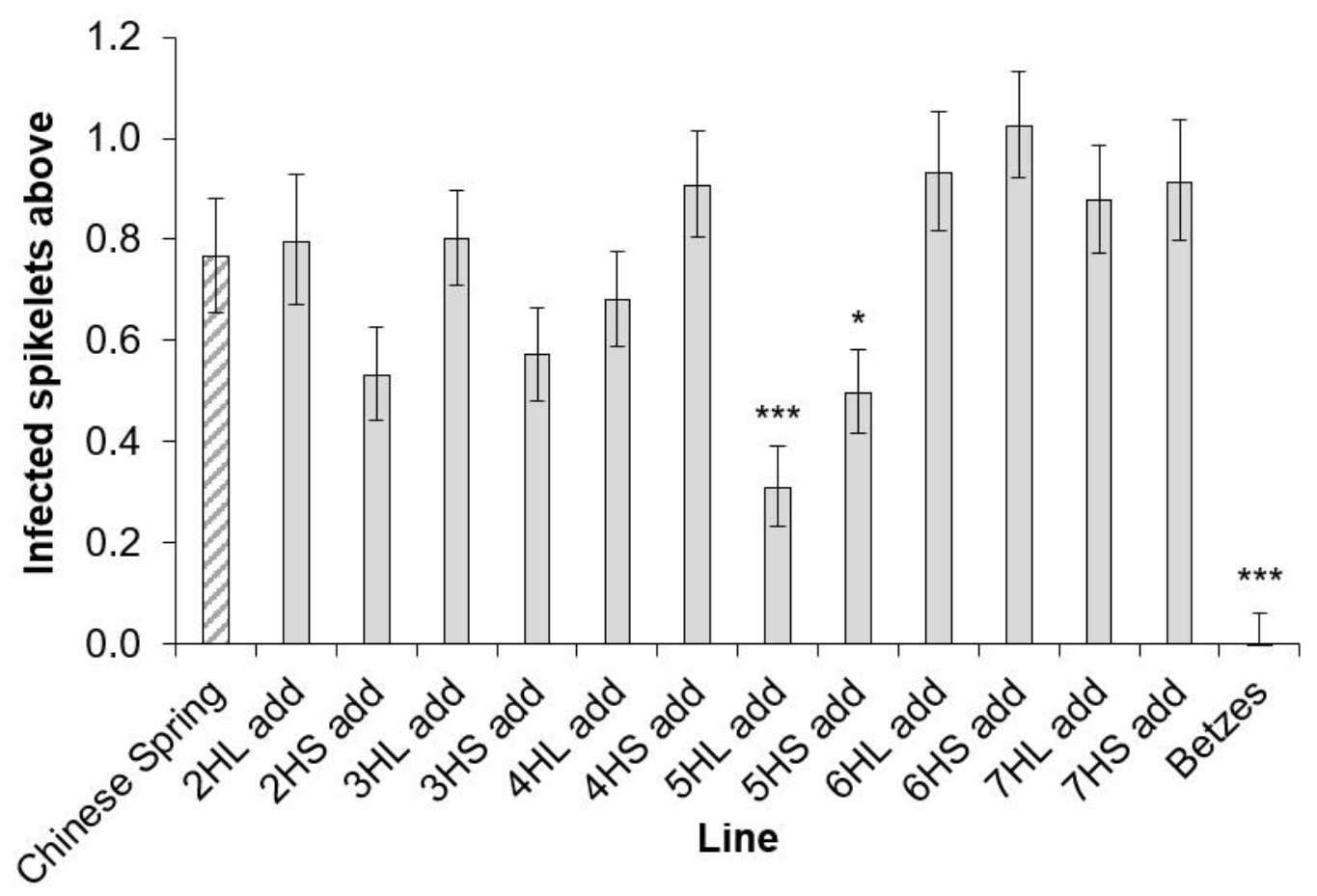




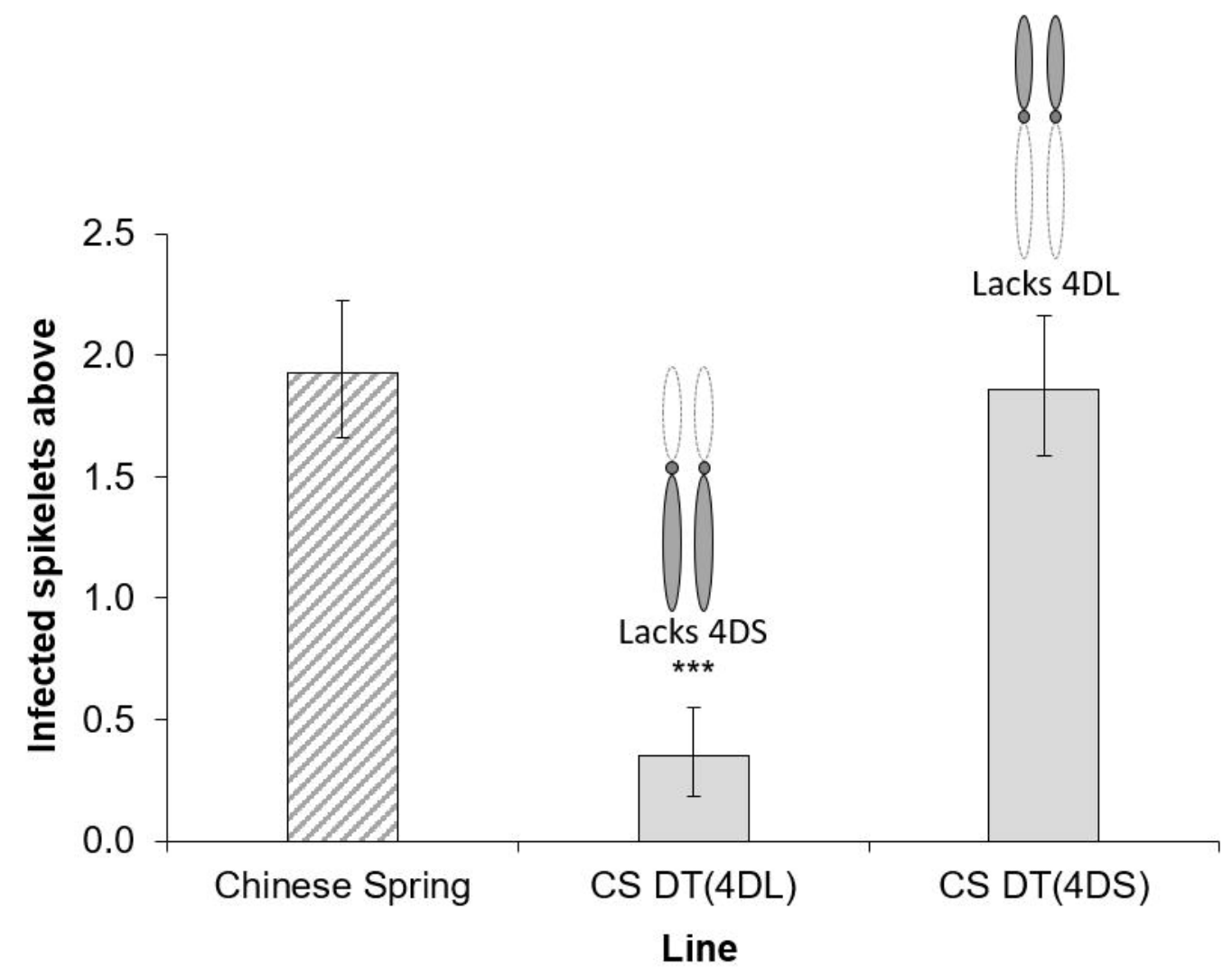


a)

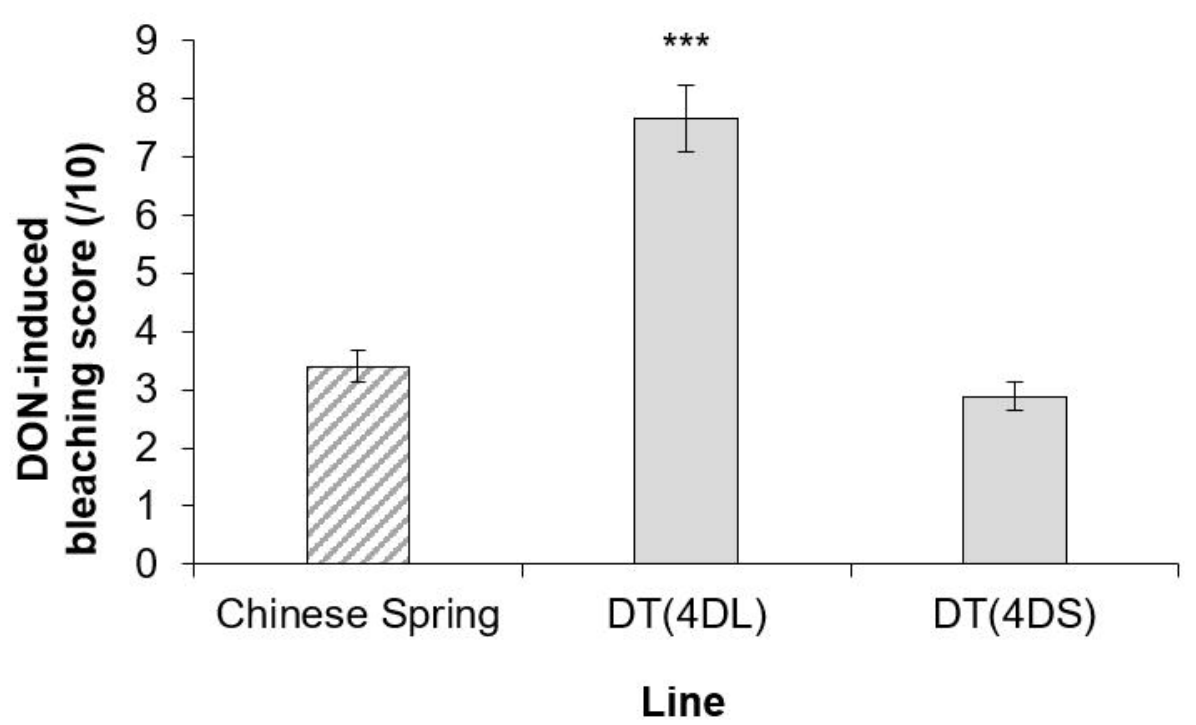

b)

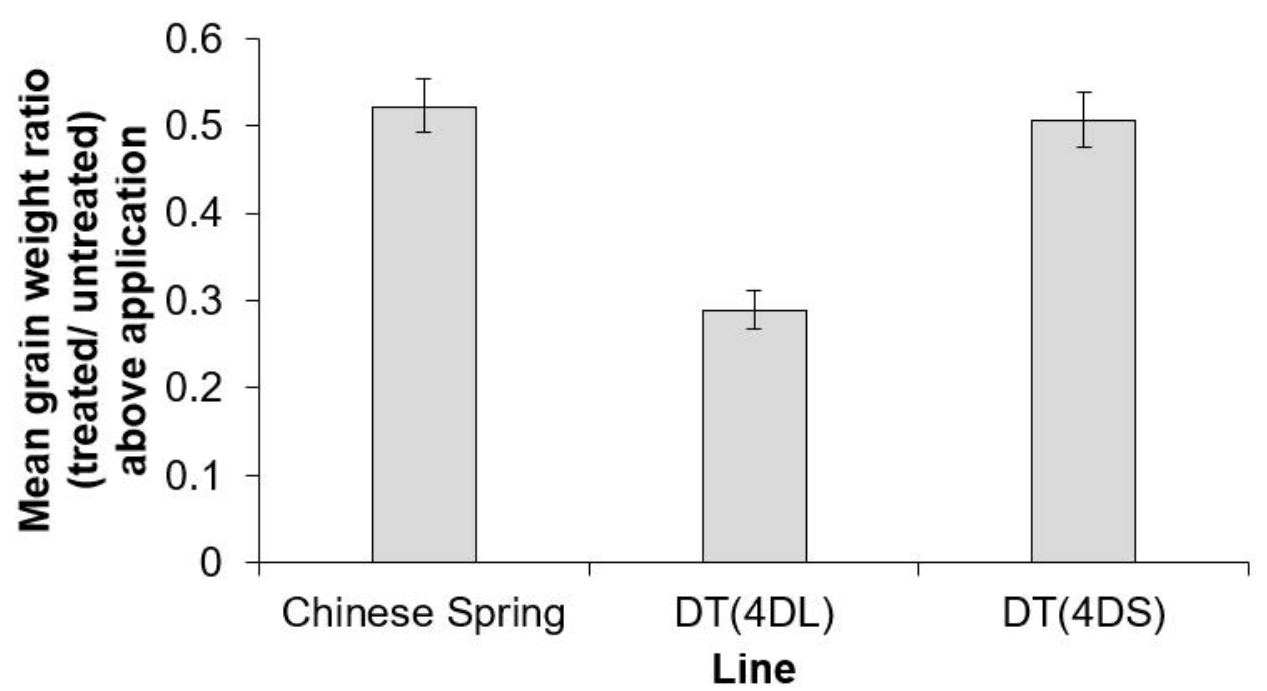

c)

Untreated

Treated

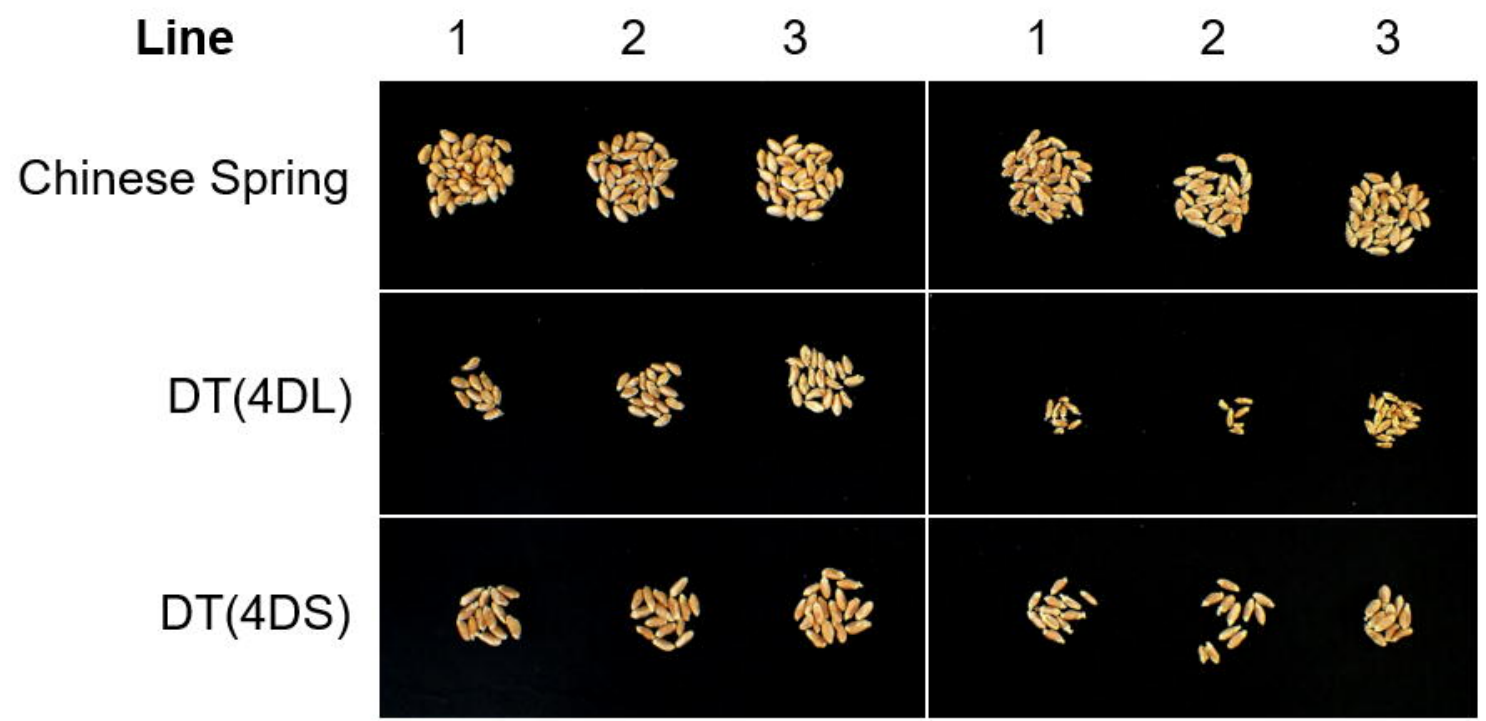


a) Chinese Spring

$\begin{array}{ll}100 & 200\end{array}$

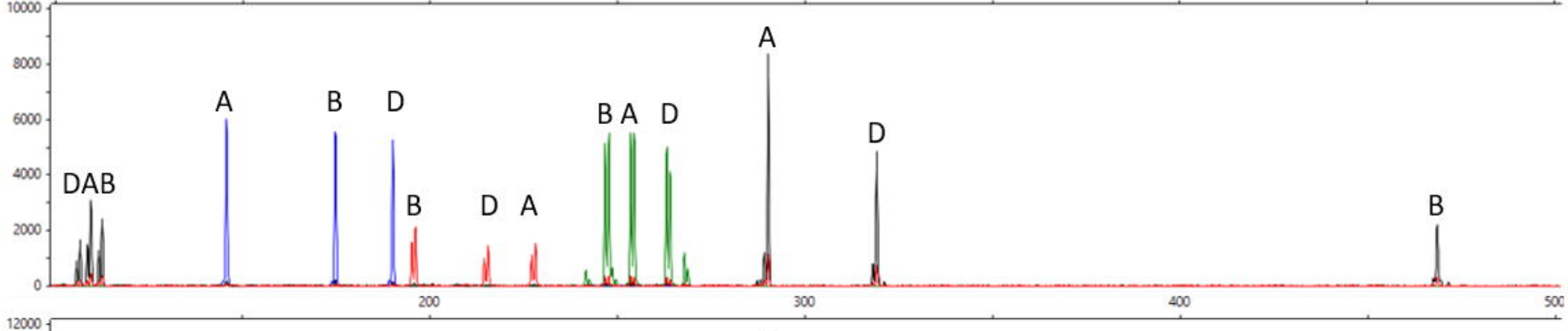

b) del4DS-2

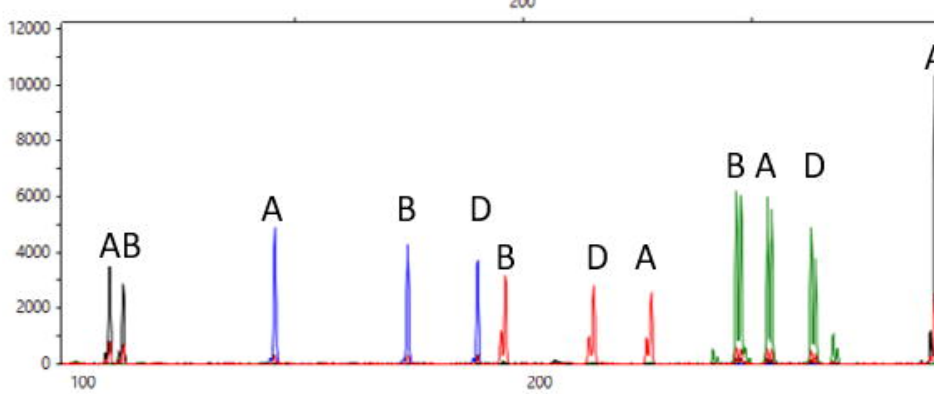

c) del4DS-4

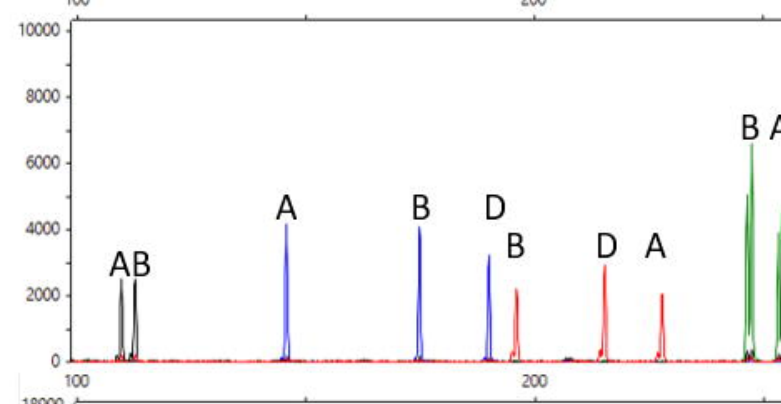

B A

d) del4DS-1

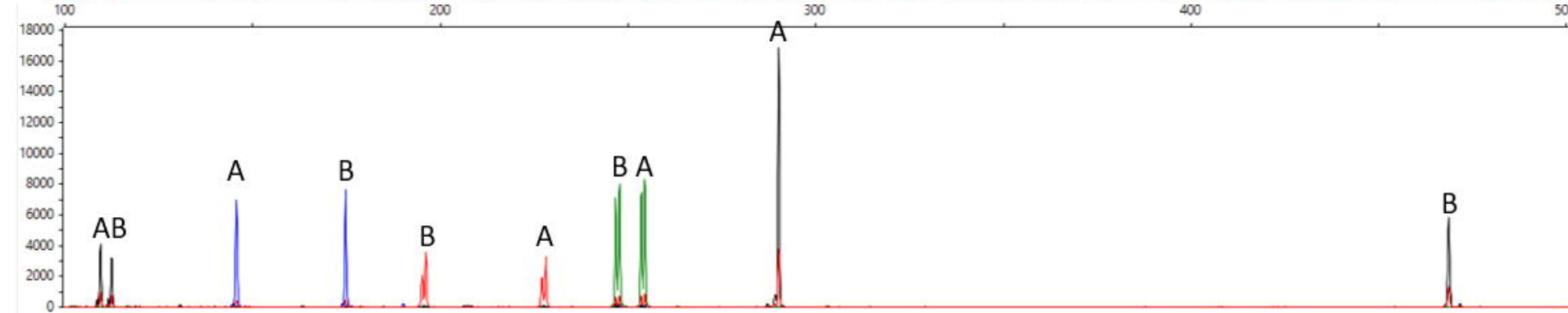




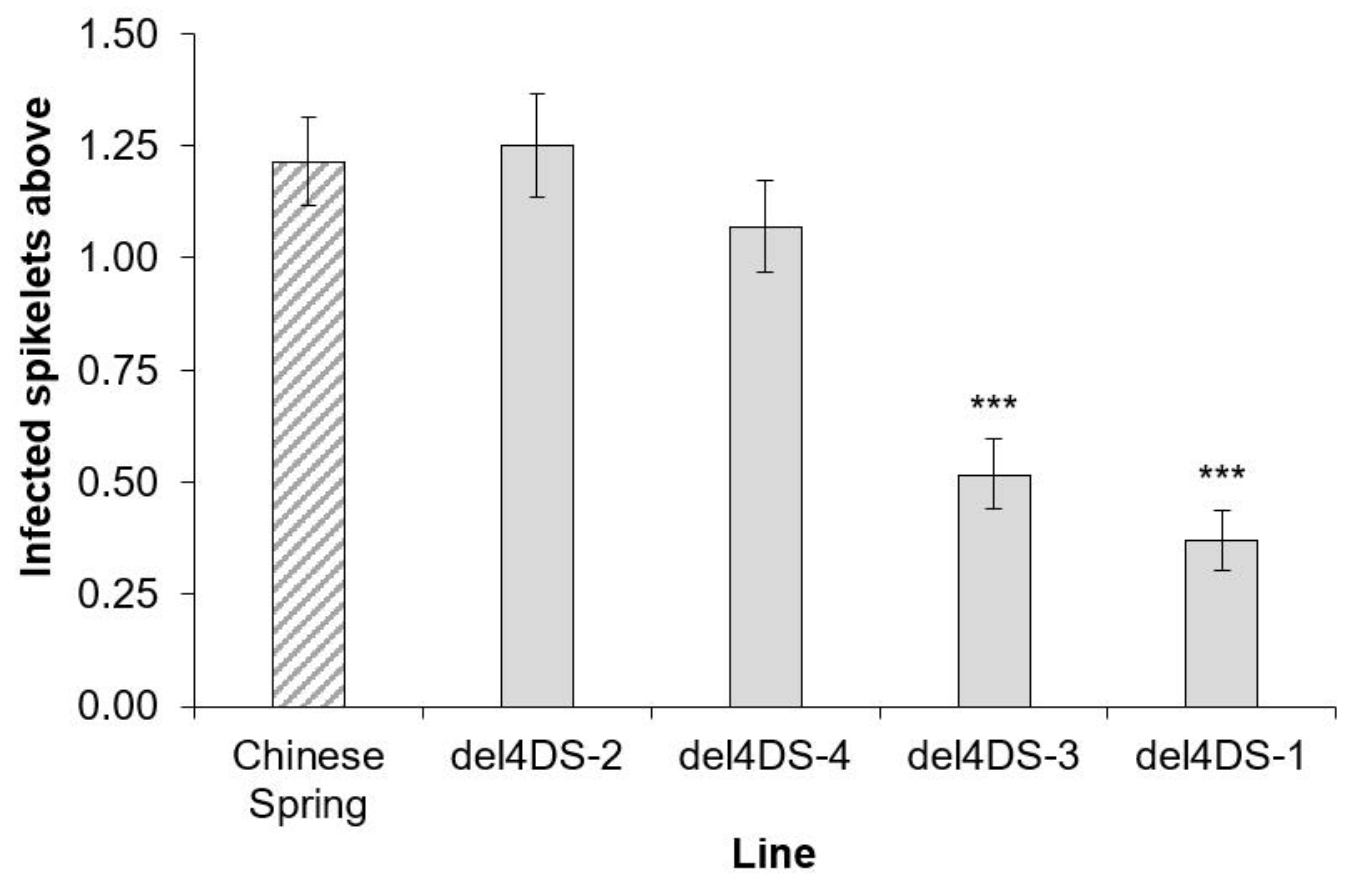




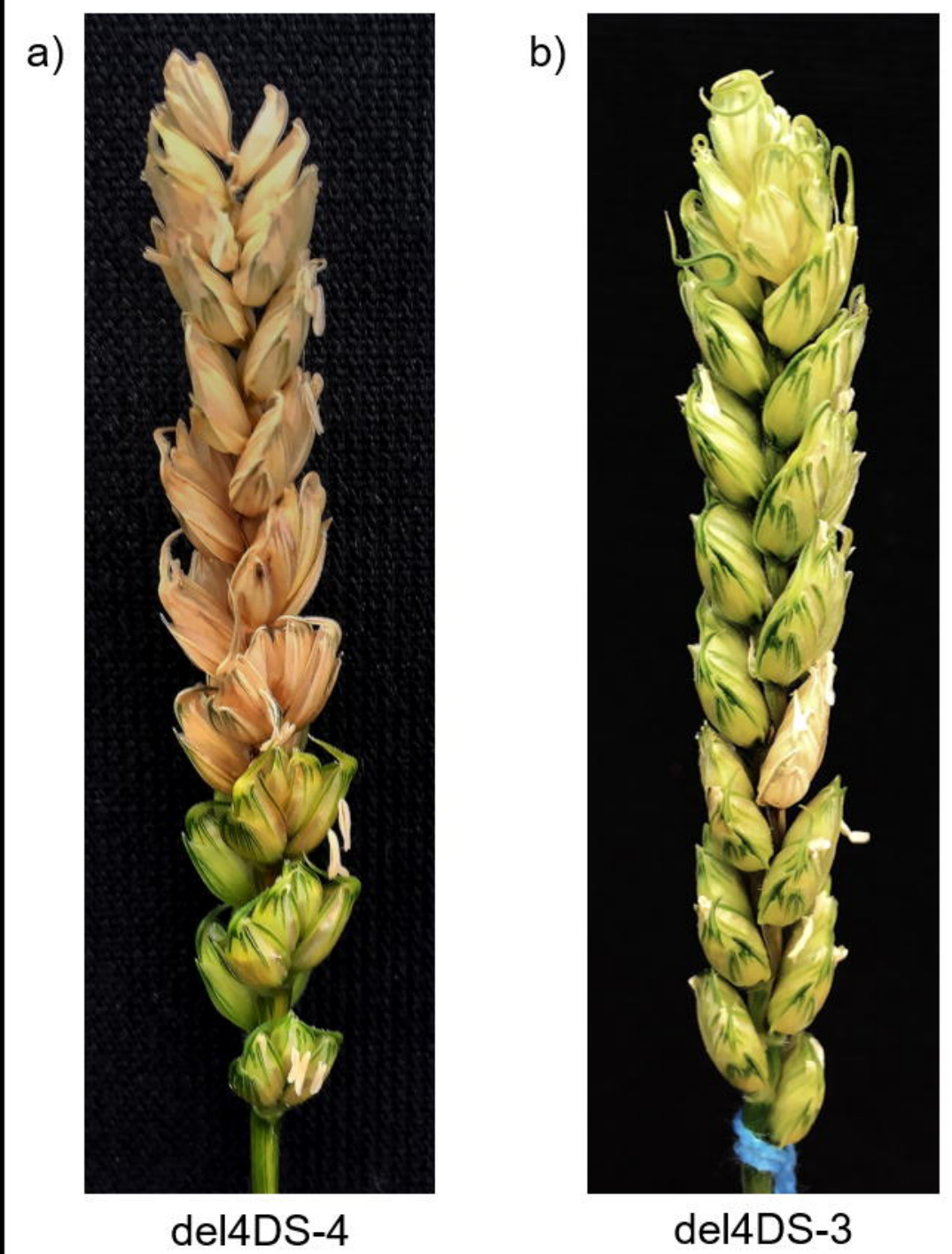


$20 \mathrm{Mbp}$

Chinese Spring Euploid

\section{del4DS-2 50.6-51.6}

$$
\text { del4DS-4 53.9-54.8 }
$$

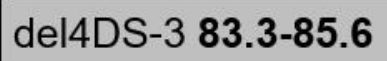

del4DS-1 111.1-140.9

Susceptibility interval 